\title{
Formation of the $\mathrm{BiAg}_{2}$ surface alloy on lattice-mismatched interfaces
}

\author{
Z. M. Abd El-Fattah, ${ }^{1,2,3, *}$ P. Lutz, ${ }^{2}$ I. Piquero-Zulaica, ${ }^{4}$ J. Lobo-Checa, ${ }^{4,5,6}$ F. Schiller, ${ }^{4}$ H. Bentmann, ${ }^{2}$ \\ J. E. Ortega, ${ }^{3,4,7}$ and F. Reinert ${ }^{2}$ \\ ${ }^{1}$ Physics Department, Faculty of Science, Al-Azhar University, Nasr City, E-11884 Cairo, Egypt \\ ${ }^{2}$ Experimentelle Physik VII and Röntgen Research Center for Complex Materials (RCCM), \\ Universität Würzburg, Am Hubland, D-97074 Würzburg, Germany \\ ${ }^{3}$ Donostia International Physics Center, Paseo Manuel Lardizabal 4, E-20018 Donostia-San Sebastián, Spain \\ ${ }^{4}$ Centro de Física de Materiales, CSIC/UPV-EHU-Materials Physics Center, Manuel Lardizabal 5, E-20018 San Sebastián, Spain \\ ${ }^{5}$ Instituto de Ciencia de Materiales de Aragón (ICMA), CSIC-Universidad de Zaragoza, E-50009 Zaragoza, Spain \\ ${ }^{6}$ Departamento de Física de la Materia Condensada, Universidad de Zaragoza, E-50009 Zaragoza, Spain \\ ${ }^{7}$ Universidad del País Vasco, Departamento de Física Aplicada I, E-20018 San Sebastián, Spain
}

(Received 25 July 2016; published 28 October 2016)

\begin{abstract}
We report on the growth of a monolayer-thick $\mathrm{BiAg}_{2}$ surface alloy on thin $\mathrm{Ag}$ films grown on $\mathrm{Pt}(111)$ and $\mathrm{Cu}(111)$. Using low energy electron diffraction (LEED), angle resolved photoemission spectroscopy (ARPES), and scanning tunneling microscopy (STM) we show that the surface structure of the $\frac{1}{3} \mathrm{ML} \mathrm{Bi} / x-\mathrm{ML} \mathrm{Ag} / \mathrm{Pt}(111)$ system $(x \geqslant 2)$ is strongly affected by the annealing temperature required to form the alloy. As judged from the characteristic $(\sqrt{3} \times \sqrt{3}) R 30^{\circ}$ LEED pattern, the $\mathrm{BiAg}_{2}$ alloy is partially formed at room temperature. A gentle, gradual increase in the annealing temperatures successively results in the formation of a pure $\mathrm{BiAg}_{2}$ phase, a combination of that phase with a $(2 \times 2)$ superstructure, and finally the pure $(2 \times 2)$ phase, which persists at higher annealing temperatures. These results complement recent work reporting the $(2 \times 2)$ as a predominant phase, and attributing the absence of $\mathrm{BiAg}_{2}$ alloy to the strained $\mathrm{Ag} / \mathrm{Pt}$ interface. Likewise, we show that the growth of the $\mathrm{BiAg}_{2}$ alloy on similarly lattice-mismatched 1 and $2 \mathrm{ML} \mathrm{Ag}-\mathrm{Cu}(111)$ interfaces also requires a low annealing temperature, whilst higher temperatures result in $\mathrm{BiAg}_{2}$ clustering and the formation of a $\mathrm{BiCu}_{2}$ alloy. The demonstration that the $\mathrm{BiAg}_{2}$ alloy can be formed on thin $\mathrm{Ag}$ films on different substrates presenting a strained interface has the prospect of serving as bases for technologically relevant systems, such as Rashba alloys interfaced with magnetic and semiconductor substrates.
\end{abstract}

DOI: 10.1103/PhysRevB.94.155447

\section{INTRODUCTION}

Heavy metal alloys, such as $\mathrm{BiAg}_{2}$, feature spin-orbit split (SOS) surface states, an essential prerequisite for the manipulation of the spin-polarized current in spintronic devices [1]. Widely explored is the control of such SOS surface states through, for example, doping with alkali metals [2], exposure to noble gases [3], or through the formation of ternary alloys where a second heavy element is added [4]. The combined interest in their surface electronic structure and the limited number of substrates where these alloys could form on top have motivated the search for heavy metal alloys in thin films grown onto different substrates [5]. For example, $\mathrm{BiAg}_{2}$ was obtained on $\mathrm{Ag}$ thin films grown onto semiconducting $\mathrm{Si}(111)$ aiming to bring the system one step closer to future technological applications [6-8]. The Ag film thickness was $\geqslant 10 \mathrm{ML}$, and hence the bulk character of the $\mathrm{Ag}$ film was already established. However, lower thicknesses are required to eliminate the strong metallic bulk contribution and to maximize possible hybridization with the underlying substrate. In $\mathrm{Bi}-\mathrm{Ag} / \mathrm{Au}(111)$ the minimum Ag film thickness that allows a well defined $(\sqrt{3} \times \sqrt{3}) R 30^{\circ}-\mathrm{BiAg}_{2}$ structure is $2 \mathrm{ML}$ [9]. The attempt to grow $\mathrm{BiAg}_{2}$ on thin $\mathrm{Ag}$ films (2-5 ML) on $\mathrm{Pt}(111)$ has been reported to form a novel $(2 \times 2)$ superstructure for a significant range of Bi coverages (0.2 ML to 0.6 ML) [10]. The authors attributed the absence of the $(\sqrt{3} \times \sqrt{3}) R 30^{\circ}$

*Corresponding author: z.m.abdelfattah@azhar.edu.eg phase to the strain at the interface between the $\mathrm{Ag}$ film and the $\mathrm{Pt}$ substrate, which is almost negligible at the $\mathrm{Ag} / \mathrm{Au}(111)$ interface. Surprisingly, the $\mathrm{BiAg}_{2}$ alloy did not form on $\mathrm{Pt}(111)$ for a $5 \mathrm{ML}$ Ag film although the LEED and ARPES data showed the $(1 \times 1)$ spots and the Shockley surface state at the clean $\operatorname{Ag}(111)$ film surface, respectively.

In this work we investigate in details the possible formation of $\mathrm{BiAg}_{2}$ alloy on such lattice-mismatched interface systems. Two different substrates are considered, namely $\operatorname{Pt}(111)$ and $\mathrm{Cu}(111)$, where the $\mathrm{Ag}$-film/substrate lattice mismatch amounts to $\sim 4 \%$ and $\sim 12 \%$, respectively. In both cases we observe the formation of the surface alloy as demonstrated by three complementary experimental techniques: low energy electron diffraction (LEED), angle resolved photoemission spectroscopy (ARPES), and scanning tunneling microscopy (STM). The characteristic $(\sqrt{3} \times \sqrt{3}) R 30^{\circ}$ superstructure and the Rashba surface state are obtained with different Ag film thickness on both substrates. In fact, the alloy formation takes place at room temperature $(300 \mathrm{~K})$, and is constrained to annealing temperatures below $(350 \pm 20) \mathrm{K}$. For the $\mathrm{Bi}$ $\mathrm{Ag} / \mathrm{Pt}(111)$ systems, higher annealing temperatures result in the formation of the previously reported $(2 \times 2)$ superstructure for Ag films with a thickness as high as $100 \mathrm{ML}$, while the annealed $\mathrm{Bi}-\mathrm{Ag} / \mathrm{Cu}(111)$ shows the formation of $\mathrm{BiCu}_{2}$ alloy at the expenses of the $\mathrm{BiAg}_{2}$ phase. These findings provide a way of growing the $\mathrm{BiAg}_{2}$ alloy and similar Rashba systems on substrates where the mismatch at the film-substrate interface might hinder their formation at reduced films thickness. 


\section{EXPERIMENTAL METHODS}

Two ARPES setups have been used: for the $\mathrm{Bi} / \mathrm{Ag} / \mathrm{Pt}(111)$ system, ARPES measurements were performed using a SCIENTA R4000 electron analyzer (Würzburg) equipped with a monochromatized He discharge lamp (MB Scientific) operating at excitation energies of $21.2 \mathrm{eV}\left(\mathrm{He}_{\alpha}\right)$ and $40.8 \mathrm{eV}$ $\left(\mathrm{He} \mathrm{II}_{\alpha}\right)$. The energy and angular resolution at $21.2 \mathrm{eV}$ were set to $\sim 5 \mathrm{meV}$ and $0.3^{\circ}$, respectively. All measurements were undertaken at $40 \mathrm{~K}$, unless specified. ARPES data on the $\mathrm{Bi} / \mathrm{Ag} / \mathrm{Cu}(111)$ system have been carried out using a SPECS Phoibos 150 electron analyzer (San Sebastián) operating with monochromatized $\mathrm{He} \mathrm{I}_{\alpha}$ photons, and with energy and angular resolution of $\sim 15 \mathrm{meV}$ and $0.3^{\circ}$, respectively. These data were collected at $150 \mathrm{~K}$. STM experiments on the $\mathrm{Bi} / \mathrm{Ag} / \mathrm{Cu}(111)$ system were taken at an Omicron VT Setup operating at $300 \mathrm{~K}$. The samples are transferred between ARPES and STM setups without breaking the UHV conditions. Both $\mathrm{Pt}(111)$ and $\mathrm{Cu}(111)$ substrates were cleaned by successive sputteringannealing cycles until sharp ARPES bands and $(1 \times 1)$ LEED spots are obtained. $\mathrm{Ag}$ and $\mathrm{Bi}$ are separately evaporated from Knudsen cells with rates of 1 and $0.06 \mathrm{ML} / \mathrm{min}$, respectively. The Ag evaporator was calibrated by depositing $1 \mathrm{ML}$ on a $\mathrm{Cu}(111)$ substrate, and annealed to $400 \mathrm{~K}$, until the corresponding gapped surface state is observed [11,12]. Likewise, a $\frac{1}{3}$ ML Bi was calibrated by $\mathrm{Bi}$ deposition onto $\mathrm{Cu}(111)$ that resulted in a sharp Rashba surface state in ARPES and a pure $(\sqrt{3} \times \sqrt{3}) R 30^{\circ}$ superstructure in LEED $[13,14]$. The Ag thin films ( $x$-ML) were first grown onto $\mathrm{Pt}(111)$ and $\mathrm{Cu}(111)$ held at $300 \mathrm{~K}$ and $150 \mathrm{~K}$ and subsequently annealed to $600 \mathrm{~K}$ and $400 \mathrm{~K}$, respectively. The $\frac{1}{3} \mathrm{ML}$ of $\mathrm{Bi}$ was then grown on top of the $x$-ML $\mathrm{Ag} / \mathrm{Pt}(111)$ and $x$-ML $\mathrm{Ag} / \mathrm{Cu}(111)$ systems at $300 \mathrm{~K}$ and $150 \mathrm{~K}$, respectively, and later annealed to different temperatures. At each annealing temperature both LEED or STM and ARPES data were acquired. Owing to the surfactant effect of $\mathrm{Bi}$, the data were found to be independent on the deposition order, i.e., equivalent results are obtained when $\mathrm{Bi}$ is grown first on both $\mathrm{Pt}(111)$ and $\mathrm{Cu}(111)$ systems followed by Ag film deposition.

\section{GROWTH OF $\frac{1}{3}$ ML Bi ON $x$-ML Ag/Pt(111)}

In Fig. 1 we present LEED patterns for a $\frac{1}{3} \mathrm{ML}$ Bi film on $\sim 3 \mathrm{ML} \mathrm{Ag} / \mathrm{Pt}(111)$. The green circles mark the $(1 \times 1)$ spots of $\mathrm{Ag} / \mathrm{Pt}(111)$ system. After Bi deposition, and without any heat treatment, a clear $\mathrm{BiAg}_{2}-(\sqrt{3} \times \sqrt{3}) R 30^{\circ}$ is formed [the unit cell is marked by the yellow lines in (a)]. Extra spots, which have been previously assigned to the $\mathrm{Bi}-(p \times \sqrt{3})$ structure [15], are also observed. Its unit cell is marked by the white lines. The coexistence of the two phases is frequent for $\mathrm{BiCu}_{2}$ and $\mathrm{BiAg}_{2}$ alloys on $\mathrm{Cu}(111)$ and $\mathrm{Ag}(111)$, respectively, when the $\mathrm{Bi}$ coverage exceeds $\frac{1}{3} \mathrm{ML}[15,16]$. In the present case, it is rather an indication of the partial alloy formation, as the deposition of the equivalent amount on $\mathrm{Cu}(111)$, taking the different lattice parameters into account, results in pure $\mathrm{BiCu}_{2}$ $(\sqrt{3} \times \sqrt{3}) R 30^{\circ}$ spots. Moreover, the extra spots completely disappear after annealing to $\sim 320 \mathrm{~K}$, leaving a sharp $\mathrm{BiAg}_{2}-$ $(\sqrt{3} \times \sqrt{3}) R 30^{\circ}$ superstructure, Fig. 1(b). After annealing to $\sim 340 \mathrm{~K}$, a partial dealloying takes place resulting in an additional $(2 \times 2)$ superstructure (red lines mark its unit cell),
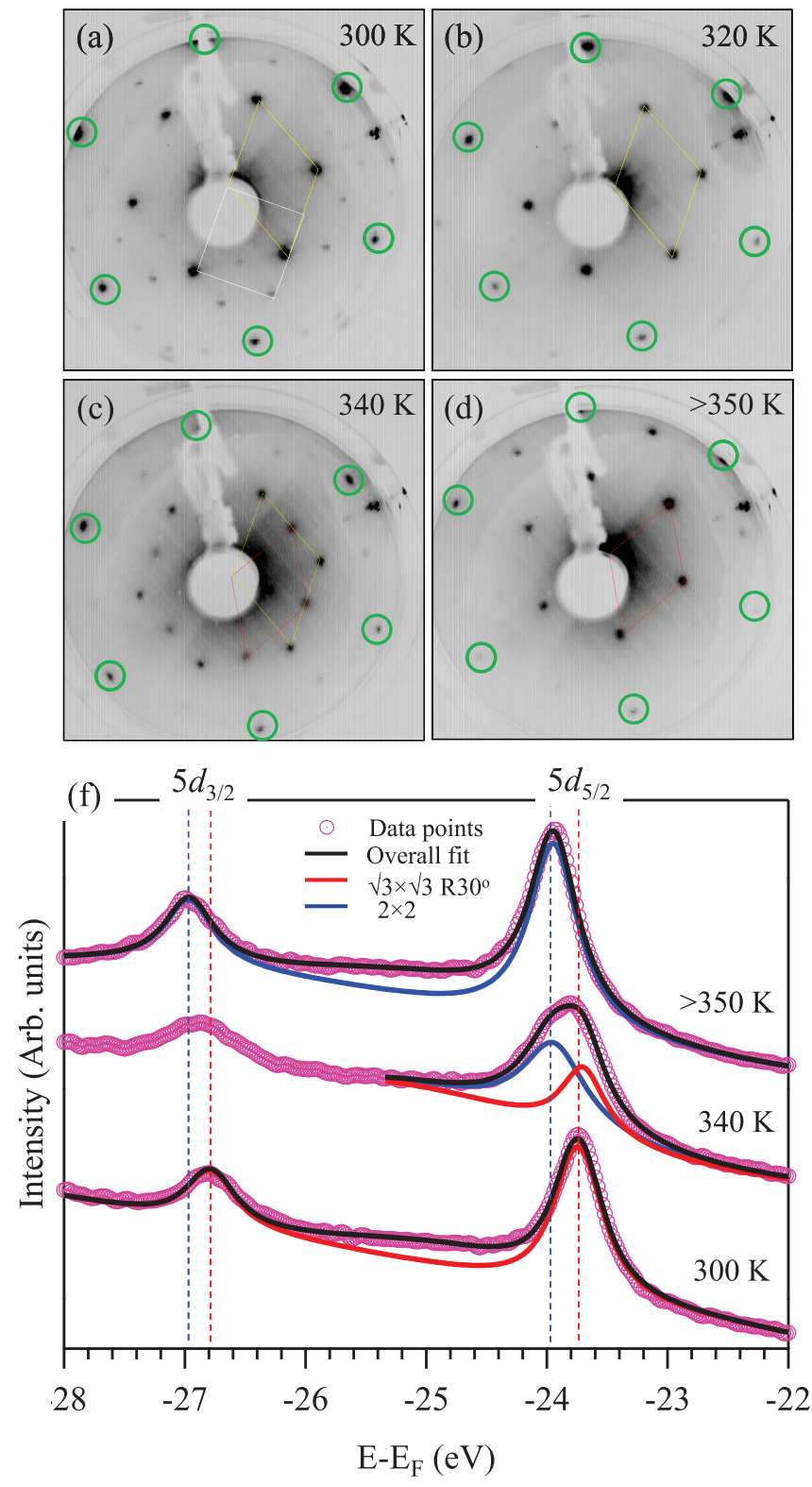

FIG. 1. (a)-(d) LEED patterns for $\frac{1}{3} \mathrm{ML}$ Bi grown on $3 \mathrm{ML}$ $\mathrm{Ag} / \mathrm{Pt}(111)$ : (a) as prepared at $300 \mathrm{~K}$, and annealed to (b) $320 \mathrm{~K}$, (c) $340 \mathrm{~K}$, and $(\mathrm{d})>350 \mathrm{~K}$. The green circles mark the $(1 \times 1)$ spots for $\mathrm{Ag} / \mathrm{Pt}(111)$. The unit cell of the $(\sqrt{3} \times \sqrt{3}) R 30^{\circ}$, the $\mathrm{Bi}-(p \times \sqrt{3})$, and the $(2 \times 2)$ are, respectively, drawn by yellow, white, and red lines. The electron energy is $(45 \pm 3) \mathrm{eV}$. (f) Photoemission spectra of the energy split Bi $5 d_{5 / 2}$ and Bi $5 d_{3 / 2}$ core levels measured with $\mathrm{He} \mathrm{II}_{\alpha}$, for $\frac{1}{3} \mathrm{ML} \mathrm{Bi}-3 \mathrm{ML} \mathrm{Ag/Pt}(111)$. The experimental spectra are displayed as purple circles, the overall fitting is denoted by solid black lines, and the red/blue curves are the peak components of the spectra and are, respectively, assigned to the $(\sqrt{3} \times \sqrt{3}) R 30^{\circ}$ and the $(2 \times 2)$ phases. Data are recorded at $300 \mathrm{~K}$.

Fig. 1(c). At higher annealing temperatures $(\geqslant 350 \mathrm{~K})$ a pure $(2 \times 2)$ superstructure is obtained, Fig. 1(d). It is important to note that the SOS alloy phase is stable within a very narrow temperature region $(<350 \mathrm{~K})$, explaining the absence of this phase in Ref. [10], where the annealing temperature was probably higher. Nonetheless, the strain is likely affecting 
the stability of the alloy, considerably reducing the annealing temperature window. We also note that the successive transition from the $(\sqrt{3} \times \sqrt{3}) R 30^{\circ}+\operatorname{Bi}-(p \times \sqrt{3})$, to the pure $(\sqrt{3} \times \sqrt{3}) R 30^{\circ}$, the mixed $(\sqrt{3} \times \sqrt{3}) R 30^{\circ}$ and $(2 \times 2)$, and finally the pure $(2 \times 2)$ superstructure is independent on the Ag-film thickness (see Supplemental Material, Fig. S1 [17]) [18-20]. However, the thicker the Ag film becomes, i.e., the lower the overall surface strain, the higher the dealloying temperature becomes.

The $(\sqrt{3} \times \sqrt{3}) R 30^{\circ}-(2 \times 2)$ structural transition could be traced by monitoring the $\mathrm{Bi} 5 d$ core level at different annealing temperatures. Figure 1(f) presents these data taken at $300 \mathrm{~K}$ with $\mathrm{He} \mathrm{II}_{\alpha}(h v=40.8 \mathrm{eV})$ for $\frac{1}{3} \mathrm{ML}$ of $\mathrm{Bi}$ on $3 \mathrm{ML}$ $\mathrm{Ag} / \mathrm{Pt}(111)$. The experimental and the overall fitting spectra are indicated with purple circles and black solid lines, respectively. The highly asymmetric background is accounted for by a Gaussian edge, made of a pure Gaussian peak on top of a linear background (see Supplemental Material, Fig. S2 [17]). For the as-deposited sample (300 K), we observed a single spin-orbit split peak (red) at binding energies of $\sim 23.74 \mathrm{eV}\left(\mathrm{Bi}-5 d_{5 / 2}\right)$ and $\sim 26.79 \mathrm{eV}\left(\mathrm{Bi}-5 d_{3 / 2}\right)$, i.e., the magnitude of the spin-orbit split amounts to $\sim 3.05 \mathrm{eV}$. Irrespective of the $(p \times \sqrt{3})-\mathrm{Bi}$ traces and the Ag film thickness, their energetic positions are always found at $(23.70 \pm 0.05) \mathrm{eV}$ and $(26.75 \pm 0.05) \mathrm{eV}$, being consistent with the reported values for the well-defined $\mathrm{BiAg}_{2}$ alloy on $\mathrm{Ag}(111)$ [21]. At $340 \mathrm{~K}$, the intensity of $\mathrm{BiAg}_{2}$ core levels decreases and a new spin-orbit split peak (blue) appears at $\sim 23.95 \mathrm{eV}\left(\mathrm{Bi}-5 d_{5 / 2}\right)$, i.e., $\sim 210 \mathrm{meV}$ shifted towards higher binding energy with respect to the energetic position obtained for the $\mathrm{BiAg}_{2}$ alloy, being now consistent with the reported value for the elemental bismuth [22,23]. This indicates a partial dealloying and the formation of the $(2 \times 2)$ superstructure reported in LEED, Fig. 1(c). At higher annealing $(\geqslant 350 \mathrm{~K})$, the spin-orbit split peak associated with the $\mathrm{BiAg}_{2}$ alloy (red) is completely suppressed, and the overall spectra could be fitted with a single spin-orbit split peak (blue), with its energetic positions at $\sim 23.95 \mathrm{eV}\left(\mathrm{Bi}-5 d_{5 / 2}\right)$ and $\sim 26.97 \mathrm{eV}\left(\mathrm{Bi}-5 d_{3 / 2}\right)$, i.e., corresponding to the $(2 \times 2)$ superstructure. The energetic position of this peak is, within $\pm 50 \mathrm{meV}$, independent of the Ag film thickness. For all cases studied, beside the characteristic shifts, the magnitude of the spin-orbit splitting is $\sim 30 \mathrm{meV}$ larger for the alloyed phase. The correspondence between LEED and the core-level photoemission data is obvious, and demonstrates the possible formation of the $\mathrm{BiAg}_{2}$ at such strained interface provided that the annealing temperature is reduced. We note that the single $\mathrm{BiAg}_{2}$ phase is hardly obtained given the limited temperature range available; nonetheless, traces of the coexisting phases do not contribute significantly to ARPES spectra [16], as we show next. We also observed that, if the deposition order is reversed, i.e., when $\mathrm{Bi}$ is deposited first then $\mathrm{Ag}$ on top, the intensity of the Bi $5 d$ levels are only weakly affected even for the as-deposited sample (see Supplemental Material, Fig. S3 [17]). This confirms the surfactant nature of $\mathrm{Bi}$, where most of the $\mathrm{Bi}$ atoms float to the surface at room temperature [10].

In the following we present ARPES data exclusively for $\mathrm{BiAg}_{2}$ alloy [i.e., after annealing to $320 \mathrm{~K}$ (a),(b), $340 \mathrm{~K}$ (c), and $400 \mathrm{~K}(\mathrm{~d})$ ] for different Ag film thickness. Figure 2 shows the Rashba SOS surface state obtained for an Ag film thickness as low as $2 \mathrm{ML}$. Thus a pair of Rashba split bands (red and blue

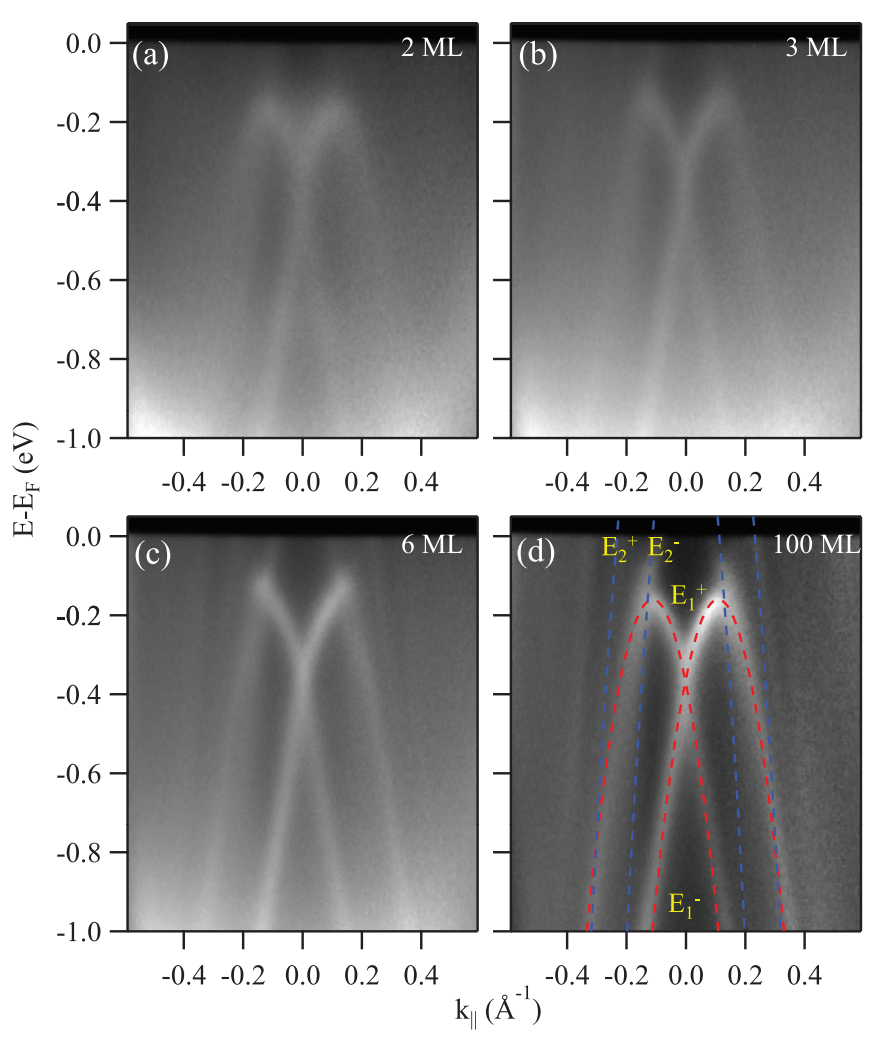

FIG. 2. Surface state band dispersion from $\frac{1}{3}$ ML $\mathrm{Bi} / x$-ML $\mathrm{Ag} / \mathrm{Pt}(111)$ after annealing to the $\mathrm{BiAg}_{2}$ formation temperatures, where (a) $x=2$, (b) $x=3$, (c) $x=6$, and (d) $x=100$. The BiAg Rashba surface state is seen in all cases with intensity enhancement for thicker films. The blue/red dashed lines assigning the Rashba bands depicted in (d) are obtained from Ref. [9] and indicated in the text.

dashed lines) termed $E_{1,2}$ are observed. Their inner and outer branches are, respectively, denoted by $E^{-}$and $E^{+}$, as indicated in Fig. 2(d) for the $100 \mathrm{ML} \mathrm{Ag} \mathrm{film} \mathrm{[9].} \mathrm{The} \mathrm{major} \mathrm{difference}$ is that the quality of the surface states is improved for higher Ag film thickness. Although the Pt(111) substrate is known to significantly alter the dispersion of the surface states of noble metal overlayers for film thickness as high as 4 ML [24,25] due to hybridization with its downward dispersing bulk $s p$ band, the behavior of the Rashba state is practically unaffected indicating its strong localization at the topmost surface layer. The blurred intensity of the surface states for reduced Ag films is likely due to the emission from the underlying bulk Pt band, in addition to the possible roughness of Bi/Ag film [see Fig. 3 on the related case of $\left.\mathrm{BiAg}_{2} / \mathrm{Ag} / \mathrm{Cu}(111)\right]$.

\section{GROWTH OF $\frac{1}{3}$ ML Bi ON $x$-ML Ag/Cu(111)}

The Ag/Pt system involves a significant interfacial strain so it is interesting to compare it to a system that a priori does not have such inherent stress, as is the $\mathrm{Ag} / \mathrm{Cu}(111)$ interface, which presents a layer-by-layer growth with moiré formation. In Fig. 3 we show STM images taken for $\frac{1}{3} \mathrm{ML} \mathrm{Bi}$ deposited at $150 \mathrm{~K}$ on (a) $1 \mathrm{ML}$ and (b) $2 \mathrm{ML} \mathrm{Ag} \mathrm{films} \mathrm{grown}$ on $\mathrm{Cu}(111)$ after gentle annealing to $350 \mathrm{~K}$ in both cases. Although the STM images for the $1 \mathrm{ML}$ and $2 \mathrm{ML} \mathrm{Ag} / \mathrm{Cu}$ 

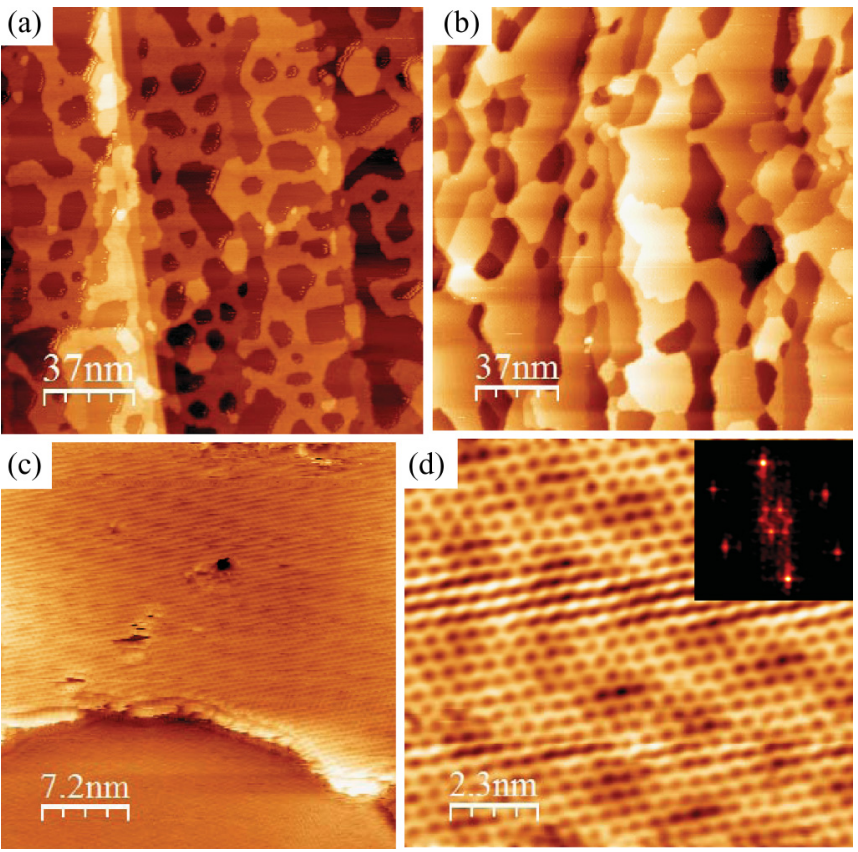

FIG. 3. Large scale STM images for $\frac{1}{3}$ ML Bi grown on (a) $1 \mathrm{ML}$ $\mathrm{Ag} / \mathrm{Cu}(111)$ and (b) $2 \mathrm{ML} \mathrm{Ag} / \mathrm{Cu}(111)$, after annealing to (350 \pm 20$)$ $\mathrm{K}$. (c) Zoom-in image of (a) revealing a moiré pattern at $\mathrm{BiAg}_{2} / \mathrm{Ag}$ islands. (d) Atomic scale image of the moiré pattern observed in (c) and its corresponding Fourier transformation of the image in the inset. The latter reveals the atomic structure of the $\mathrm{BiAg}_{2}$ alloy (outer large hexagon) and the moiré pattern (inner small hexagon) and their relative orientation $\left(\sim 30^{\circ}\right.$ rotated $)$.

overlayer systems prior to Bi deposition exhibit a perfect layer-by-layer growth (see Supplemental Material, Fig. S4 [17]), the Bi deposition roughens the surface, giving rise to $\mathrm{BiAg}_{2} / \mathrm{Ag}$ islands (bright areas) and $\mathrm{Ag}$-free areas (dark areas). The $\mathrm{BiAg}_{2}$-covered areas amount to $\sim 50 \%$ and $\sim 85 \%$ for the $1 \mathrm{ML} \mathrm{Ag}$ and $2 \mathrm{ML} \mathrm{Ag}$, respectively, whereas the lateral dimension of the islands is clearly larger for $2 \mathrm{ML} \mathrm{Ag}$. In both cases the height of the majority of the islands is $\sim 4.6 \AA$, which correspond to bilayer $\mathrm{BiAg}_{2} / \mathrm{Ag}$ islands. Thicker islands are also observed, which make up for the total 2 ML Ag coverage. On the other hand, excess Bi may begin to alloy with $\mathrm{Cu}$, at apparently structureless $\mathrm{Cu}$ areas (see discussion below). A zoom-in image of Fig. 3(a) is shown in Fig. 3(c). The image contains two distinct areas, the Ag-free structureless area and the bilayer $\mathrm{BiAg}_{2} / \mathrm{Ag}$ region exhibiting a moiré-like pattern. Such a moiré superstructure is characteristic for Bi-free, $2 \mathrm{ML} \mathrm{Ag} / \mathrm{Cu}(111)$, although the same moiré pattern may also be expected for the $\mathrm{BiAg}_{2} / \mathrm{Ag} / \mathrm{Cu}(111)$ bilayer, as alloying with $\mathrm{Bi}$ does not change the $\mathrm{Ag}-\mathrm{Ag}$ nearest neighbor distance (see Supplemental Material, Fig. S5 [17]). Both the moiré periodicity and the atomic lattice parameter can be deduced from the atomic scale STM image shown in (d). The moiré periodicity is found to be the same as for the $2 \mathrm{ML} \mathrm{Ag/Cu}$ (i.e., $\sim 24 \AA$ ), whereas the atomic scale resolution reveals the characteristic $\mathrm{BiAg}_{2}-(\sqrt{3} \times \sqrt{3}) R 30^{\circ}$ with a lattice parameter of $5.1 \AA$ [26]. Furthermore, the moire pattern is $\sim 30^{\circ}$-rotated with respect to the atomic lattice, as shown in the Fourier transform at the image's inset.
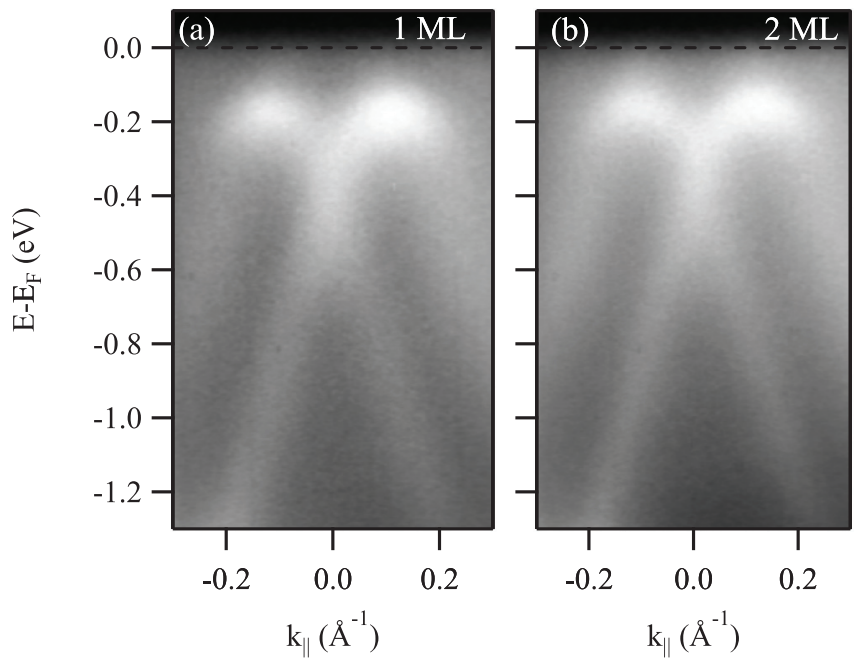

FIG. 4. ARPES photoemission intensity plot after annealing to $(350 \pm 20) \mathrm{K}$ for $\frac{1}{3} \mathrm{ML}$ of $\mathrm{Bi}$ on (a) $1 \mathrm{ML}$ and (b) $2 \mathrm{ML} \mathrm{Ag/Cu(111)}$ The dispersion relations in both cases are identical, except that the slightly sharper bands in (b) can be associated to the larger lateral extent of the bilayer Ag islands compared to (a).

In Fig. 4 the ARPES photoemission intensity plots for the $\frac{1}{3} \mathrm{ML} \mathrm{Bi}$ on $1 \mathrm{ML}$ and $2 \mathrm{ML} \mathrm{Ag}$ annealed to $350 \mathrm{~K}$ are presented. A distinct $\mathrm{BiAg}_{2}$ Rashba surface state is obtained for both Ag coverages. Only a relative improvement in the quality of the surface states is visible, i.e., sharper bands are obtained for the Bi-2 ML Ag sample, as expected for the larger lateral extent of the $\mathrm{BiAg}_{2}$ bilayers in Fig. 3(b). In fact, the quality of the surface states is comparable to the $\mathrm{Bi} / 2-3$ ML $\mathrm{Ag} / \mathrm{Pt}(111)$ presented in Figs. 2(a) and 2(b), indicating that the reduced quality for both systems is related to surface roughness and overall island size. Such a growth behavior is likely triggered by both the well-known high mobility of Ag atoms (kinetics) and the interface mismatch (energetics). The latter is negligible at $\mathrm{Ag} / \mathrm{Au}(111)$ interface and, therefore, $\frac{1}{3} \mathrm{ML} \mathrm{Bi}$ on $1 \mathrm{ML} \mathrm{Ag} / \mathrm{Au}(111)$ exhibits no $\mathrm{BiAg}_{2}$ phase, indicating that a minimum of one $\mathrm{Ag}$ bilayer is required for the alloy formation. Another important similarity between the two systems is that the exclusive $\mathrm{BiAg}_{2}$ alloy phase is only obtained at low annealing temperatures $(350 \mathrm{~K})$; however, the behavior at higher annealing is very different between both substrates, as we show next.

In Fig. 5 we show ARPES data for a $\frac{1}{3}$ ML Bi grown on $1 \mathrm{ML} \mathrm{Ag} / \mathrm{Cu}(111)$ at $150 \mathrm{~K}$, and postannealed to (a) $430 \mathrm{~K}$ and to (b),(c) $600 \mathrm{~K}$. The $\mathrm{BiCu}_{2}$ surface state can be identified in both cases [3], while the $\mathrm{BiAg}_{2}$ shifts toward higher binding energy. Its Dirac point is now found at $(-520 \pm 10)$ $\mathrm{meV}$. It is only at $430 \mathrm{~K}$, where the $\mathrm{BiAg}_{2}$ surface state intensity is comparable to the $\mathrm{BiCu}_{2}$, which can be mistaken with the $E_{2}^{-}$state coming from the unoccupied region. This indicates that the dark areas in Fig. 3(a) are nonalloyed $\mathrm{Bi} / \mathrm{Cu}$ which transform into $\mathrm{BiCu}_{2}$ alloy upon annealing. The formation of $\mathrm{BiCu}_{2}$ alloy is further justified by measuring its characteristic lattice parameter, $\sim 4.4 \AA$, in atomically resolved STM images (see Supplemental Material, Fig. S6 [17]). Above this temperature, ARPES data consists mainly of $\mathrm{BiCu}_{2}$ and very weak $\mathrm{BiAg}_{2}$ bands. This mixture of $\mathrm{BiCu}_{2}$ and weak 

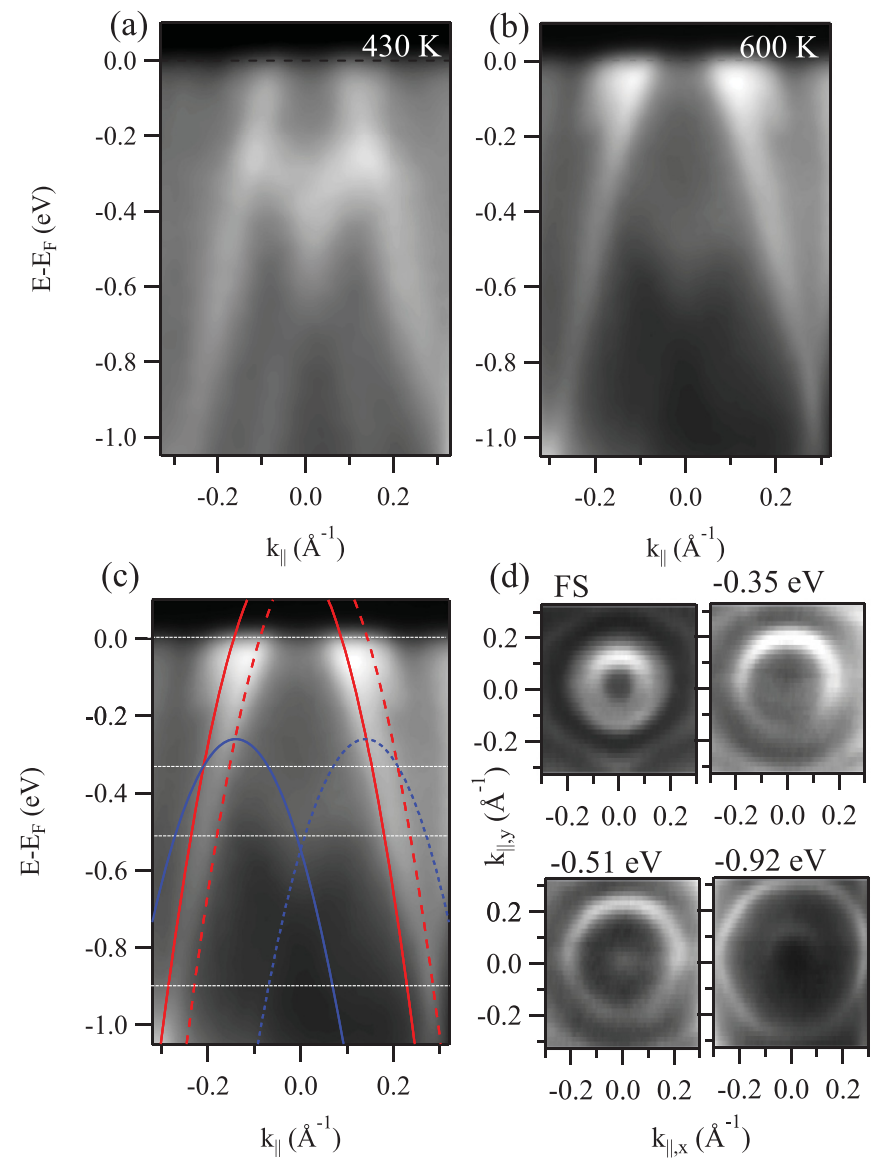

FIG. 5. (a),(b) Photoemission intensity plot for $\frac{1}{3}$ ML Bi on $1 \mathrm{ML} \mathrm{Ag} / \mathrm{Cu}(111)$ after annealing to (a) $430 \mathrm{~K}$ and (b) $600 \mathrm{~K}$. BiCu and $\mathrm{BiAg}_{2}$ surface states coexist with the weight of $\mathrm{BiAg}_{2}$ decreasing upon annealing. In both cases the $\mathrm{BiAg}_{2}$ surface state shifts towards higher binding energy compared to the $350 \mathrm{~K}$ annealed sample. (c) The same photoemission intensity plot shown in (b) overlaid with the characteristic surface state bands for pure $\mathrm{BiCu}_{2}$ (red lines) and $\mathrm{BiAg}_{2}$ (blue lines). The dispersion parameters are the ones reported for $\mathrm{BiCu}_{2} / \mathrm{Cu}(111)$ and $\mathrm{BiAg}_{2} / \mathrm{Ag}(111)$ except that the latter is shifted towards higher binding energy. (d) Constant energy surfaces taken at the indicated energies (white lines) in (c).

$\mathrm{BiAg}_{2}$ surface states is stable up to annealing temperatures of $800 \mathrm{~K}$. The strongly reduced intensity of the $\mathrm{BiAg}_{2}$ surface states clearly indicates a dominant clustering and dealloying at such temperatures. In Fig. 5(c) we overlay the photoemission intensity in (b) with the parabolic dispersion reported for $\mathrm{BiCu}_{2}$ (red lines) and $\mathrm{BiAg}_{2}$ (blue lines) surface states. The red lines coincide with $\mathrm{BiCu}_{2}$ bands, whereas the blue lines are shifted by $110 \mathrm{meV}$ to higher binding energy. The coexistence of the two surface states can also be seen in the constant energy surfaces (CES) taken at the white dashed lines in (c) and presented in (d). The Fermi surface (FS) cut shows the three contours for $\mathrm{BiCu}_{2}$, the $-350 \mathrm{meV}$ cut shows the main $\mathrm{BiCu}_{2}$ contour with some inner intensity from the $\mathrm{BiAg}_{2}$, the $-510 \mathrm{meV}$ cut shows the main $\mathrm{BiCu}_{2}$ bands and the Dirac point of the $\mathrm{BiAg}_{2}$, and the $-920 \mathrm{meV}$ cut shows the $\mathrm{BiCu}_{2}$ (outer) and $\mathrm{BiAg}_{2}$ (inner) rings. The reduced intensity and the shift of the $\mathrm{BiAg}_{2}$ surface state to higher binding energy are likely associated with the presence of two-dimensional $\mathrm{BiAg}_{2}$ crystallites of very small dimensions (see Supplemental Material, Fig. S6 [17]) [27].

\section{CONCLUSION}

In conclusion, we have shown that it is possible to form the $\mathrm{BiAg}_{2}$ alloy for ultrathin $\mathrm{Ag}$ films, even on very strained interfaces. Indeed, the structure of a $\frac{1}{3} \mathrm{ML} B \mathrm{~B}$ on thin $\mathrm{Ag}$ films grown on $\mathrm{Pt}(111)$ and $\mathrm{Cu}(111)$ is strongly affected by the annealing temperature. For both substrates, the formation of the $\mathrm{BiAg}_{2}$ alloy is only possible for annealing temperatures lower than $(350 \pm 20) \mathrm{K}$. The electronic structure of the $\mathrm{BiAg}_{2}$ surface alloy, in both systems, was found to be qualitatively similar to $\mathrm{BiAg}_{2} / \mathrm{Ag} / \mathrm{Au}(111)$. At higher annealing temperatures, the previously reported $(2 \times 2)$ superstructure is obtained on $\mathrm{Bi} / \mathrm{Ag} / \mathrm{Pt}(111)$ independent on the $\mathrm{Ag}$ film thickness. However, higher annealing temperatures on $\mathrm{Bi} / \mathrm{Ag} / \mathrm{Cu}(111)$ systems result in the formation of $\mathrm{BiCu}_{2}$ in combination with $\mathrm{BiAg}_{2}$ traces.

The finding that the formation of $\mathrm{BiAg}_{2}$ surface alloy on strained interfaces is limited to a narrow temperature range should have some implications on the search for $\mathrm{BiAg}_{2}$ and similar Rashba alloys on technologically relevant substrates. For example, Ag grows in a layer-by-layer mode on $\mathrm{Ni}(111)$, although the lattice mismatch is larger than that for both $\mathrm{Cu}(111)$ and $\mathrm{Pt}(111)$. $\mathrm{Ni}(111)$ supports spin-polarized electronic states and therefore the interplay between Rashba and exchange interactions could be investigated [28,29]. Although the $\mathrm{BiAg}_{2}$ alloy has been previously grown on $\mathrm{Si}(111)$ [6-8], the Ag film thickness could be lowered and a Rashba surface state interface with a semiconductor substrate could be obtained.

\section{ACKNOWLEDGMENTS}

We would like to acknowledge Dr. Jens Brede for his help during STM data acquisition. This work was supported by the Spanish Gouvernment (Grant No. MAT2013-46593-C6-4-P), the Basque Gouvernment (Grant No. IT621-13), and the Spanish Research Council (Grant No. CSIC-201560I022). Z.M.A. would like to acknowledge funding from DAAD and DIPC. P.L. would also like to acknowledge funding from the Deutsche Forschungsgemeinschaft via Project No.RE 1469/8-1.
[1] C. R. Ast, J. Henk, A. Ernst, L. Moreschini, M. C. Falub, D. Pacilé, P. Bruno, K. Kern, and M. Grioni, Phys. Rev. Lett. 98, 186807 (2007).

[2] E. Frantzeskakis, A. Crepaldi, S. Pons, K. Kerna, and M. Grioni, J. Electron Spectrosc. Relat. Phenom. 181, 88 (2010).

[3] H. Bentmann and F. Reinert, New J. Phys. 15, 115011 (2013).
[4] I. Gierz, F. Meier, J. H. Dil, K. Kern, and Ch. R. Ast, Phys. Rev. B 83, 195122 (2011).

[5] A. C. Potter and P. A. Lee, Phys. Rev. Lett. 105, 227003 (2010).

[6] K. He, T. Hirahara, T. Okuda, S. Hasegawa, A. Kakizaki, and I. Matsuda, Phys. Rev. Lett. 101, 107604 (2008). 
[7] E. Frantzeskakis, S. Pons, H. Mirhosseini, J. Henk, C. R. Ast, and M. Grioni, Phys. Rev. Lett. 101, 196805 (2008).

[8] A. Crepaldi, S. Pons, E. Frantzeskakis, K. Kern, and M. Grioni, Phys. Rev. B 85, 075411 (2012).

[9] H. Bentmann, S. Abdelouahed, M. Mulazzi, J. Henk, and F. Reinert, Phys. Rev. Lett. 108, 196801 (2012).

[10] E. Frantzeskakis, S. Pons, A. Crepaldi, H. Brune, K. Kern, and M. Grioni, Phys. Rev. B 84, 245443 (2011).

[11] F. Schiller, J. Cordón, D. Vyalikh, A. Rubio, and J. E. Ortega, Phys. Rev. Lett. 94, 016103 (2005).

[12] A. Bendounan, F. Forster, J. Ziroff, F. Schmitt, and F. Reinert, Phys. Rev. B 72, 075407 (2005).

[13] H. Bentmann, F. Forster, G. Bihlmayer, E. V. Chulkov, L. Moreschini, M. Grioni, and F. Reinert, Europhys. Lett. 87, 37003 (2009).

[14] L. Moreschini, A. Bendounan, H. Bentmann, M. Assig, K. Kern, F. Reinert, J. Henk, C. R. Ast, and M. Grioni, Phys. Rev. B 80, 035438 (2009).

[15] K. H. Zhang, I. M. McLeod, M. Lahti, K. Pussi, and V. R. Dhanak, J. Phys.: Condens. Matter 24, 435502 (2012).

[16] L. Moreschini, A. Bendounan, C. R. Ast, F. Reinert, M. Falub, and M. Grioni, Phys. Rev. B 77, 115407 (2008).

[17] See Supplemental Material at http://link.aps.org/supplemental/ 10.1103/PhysRevB.94.155447 for additional information on the $\mathrm{Bi} / \mathrm{Ag} / \mathrm{Pt}$ system at reduced $\mathrm{Ag}$ thickness, the fitting of core-level data, the surfactant property of Bismuth, the film roughness induced by $\mathrm{Bi}$ deposition, the $\mathrm{BiAg}_{2}$ moiré pattern, and the simultaneous formation of $\mathrm{BiCu}_{2}$ surface alloy and $\mathrm{BiAg}_{2}$ nanocrystallite.

[18] One exception is the $\sim 2$ ML Ag case, where, in addition to the $(\sqrt{3} \times \sqrt{3}) R 30^{\circ}$ and the $(2 \times 2)$ spots in Fig. 1(c), traces of the
$(4 \times 4)$ superstructure spots were detected. In fact these extra spots could be due $\mathrm{Bi} / \mathrm{Pt}(111)$ areas present at such a reduced thickness.

[19] M. T. Paffett, C. T. Campbell, and T. N. Taylor, J. Chem. Phys. 85, 6176 (1986).

[20] Z. M. Abd El-Fattah, P. Lutz, H. Maaß, H. Bentmann, and F. Reinert (unpublished).

[21] H. Bentmann, H. Maaß, T. R. F. Peixoto, C. Seibel, M. Leandersson, T. Balasubramanian, P. Krüger, and F. Reinert, arXiv:1507.04664.

[22] A. V. Naumkin, A. Kraut-Vass, S. W. Gaarenstroom, and C. J. Powell, NIST X-ray Photoelectron Spectroscopy Database (http://srdata.nist.gov/xps/Default.aspx).

[23] M. T. Edmonds, J. T. Hellerstedt, A. Tadich, A. Schenk, K. M. ODonnell, J. Tosado, N. P. Butch, P. Syers, J. Paglione, and M. S. Fuhrer, J. Phys. Chem. C 118, 20413 (2014).

[24] P. Moras, D. Wortmann, G. Bihlmayer, L. Ferrari, G. Alejandro, P. H. Zhou, D. Topwal, P. M. Sheverdyaeva, S. Blügel, and C. Carbone, Phys. Rev. B 82, 155427 (2010).

[25] P. Moras, P. M. Sheverdyaeva, C. Carbone, D. Topwal, L. Ferrari, G. Bihlmayer, S. Ouazi, S. Rusponi, A. Lehnert, and H. Brune, J. Phys.: Condens. Matter 24, 335502 (2012).

[26] C. Kato, Y. Aoki, and H. Hirayama, Phys. Rev. B 82, 165407 (2010).

[27] G. Vasseur, J. Lobo-Checa, I. Piquero, J. Brede, and J. E. Ortega, (private communication, 2016).

[28] J. Lobo-Checa, T. Okuda, M. Hengsberger, L. Patthey, T. Greber, P. Blaha, and J. Osterwalder, Phys. Rev. B 77, 075415 (2008).

[29] K. Aït-Mansour and O. Gröning, Surf. Sci. 604, 872 (2010). 